\title{
A Protocol of Process Evaluation of Interventions for the Prevention of Type 2 Diabetes in Women with Gestational Diabetes Mellitus: A Systematic Review
}

Iklil Iman Mohd Sa'id

Universiti Putra Malaysia https://orcid.org/0000-0002-7330-9397

Iliatha Papachristou Nadal

King's College London

Angus Forbes

King's College London

Kimberley Goldsmith

King's College London

Irmi Zarina Ismail

Universiti Putra Malaysia

Faezah Hassan

Universiti Putra Malaysia

Siew Mooi Ching

Universiti Putra Malaysia

Nicola Guess

University of Westminster

Helen Murphy

King's College London

Matthew Prina

King's College London

Barakatun Nisak Mohd Yusof

Universiti Putra Malaysia

Nurul Iftida Basri

Universiti Putra Malaysia

Mazatulsafura Sf Salim

Universiti Putra Malaysia

Nur Hafizah Mahamad Sobri

Universiti Putra Malaysia

Choiriyatul 'Azmiyaty Amar Ma'ruf

Universiti putra malaysia 


\section{Pamela Phui Har Yap}

Universiti Putra Malaysia

Khalida Ismail

King's College London

Boon How Chew ( $\nabla$ chewboonhow@upm.edu.my)

Universiti Putra Malaysia https://orcid.org/0000-0002-8627-6248

\section{Protocol}

Keywords: Process evaluations, gestational diabetes mellitus, randomized controlled trial, diabetes prevention, lifestyle intervention, maternal outcomes, and antenatal outcomes

Posted Date: November 2nd, 2020

DOI: https://doi.org/10.21203/rs.3.rs-99446/v1

License: (c) (1) This work is licensed under a Creative Commons Attribution 4.0 International License. Read Full License 


\section{A protocol of process evaluation of interventions for the prevention of type 2 diabetes}

\section{in women with gestational diabetes mellitus: a systematic review.}

\section{Authors}

Iklil Iman Mohd Sa'id ${ }^{1}$, Iliatha Papachristou $\mathrm{Nadal}^{2}$, Angus Forbes ${ }^{3}$, Kimberley Goldsmith ${ }^{5}$, Irmi Zarina Ismail ${ }^{1}$, Faezah Hassan ${ }^{1}$, Ching Siew Mooi ${ }^{1}$, Nicola Guess ${ }^{6}$, Helen Murphy ${ }^{7}$, Matthew Prina ${ }^{8}$, Barakatun Nisak Mohd Yusoff ${ }^{9}$, Nurul Iftida Basri ${ }^{10}$, Mazatulfazura Sf Binti Salim ${ }^{11}$,Nur Hafizah Mahamad Sobri ${ }^{1}$, Choiriyatul Azmiyaty ${ }^{1}$, Pamela Phui Har Yap ${ }^{1}$, Khalida Ismail ${ }^{4}$, Boon How Chew ${ }^{1}$. On behalf of MYGODDESS Project team.

\section{Affiliations}

${ }^{1}$ Department of Family Medicine, Faculty of Medicine and Health Sciences, Universiti Putra Malaysia, 43400 Serdang, Selangor, Malaysia.

${ }^{2}$ King's College London/London School of Hygiene and Tropical Medicine

${ }^{3}$ Care of Long-term Conditions Division, King's College London.

${ }^{4}$ Dept of Psychological Medicine, King's College London

${ }^{5}$ Institute of Psychiatry, Psychology \& Neuroscience, King's College London

${ }^{6}$ Research Centre for Optimal Health, University of Westminster, London, UK.

${ }^{7}$ Norwich Medical School, Bob Champion Research and Education Building, University of East Anglia, Norwich, UK/Division of Women's Health, St Thomas' campus, Kings College London, UK/Honorary Consultant Physician, Elsie Bertram Diabetes Centre, Norfolk and Norwich University Hospital, UK.

${ }^{8}$ Department of health service and population research, Institute of Psychiatry, Psychology and Neuroscience, King's College London

${ }^{9}$ Department of Dietetics, Faculty of Medicine and Health Sciences, Universiti Putra Malaysia, 43400 Serdang, Selangor, Malaysia.

${ }^{10}$ Department of Obstetrics and Gynaecology, Faculty of Medicine and Health Sciences, Universiti Putra Malaysia, 43400 Serdang, Selangor, Malaysia. 
${ }^{11}$ Department of Rehabilitation Medicine, Faculty of Medicine and Health Sciences, Universiti Putra Malaysia, 43400 Serdang, Selangor, Malaysia.

\section{Corresponding author}

Boon-How Chew, Department of Family Medicine, Faculty of Medicine and Health Sciences, Universiti Putra Malaysia, 43400 Serdang, Selangor, Malaysia.

Email: chewboonhow@upm.edu.my. 


\section{Word count: 348}

\section{Abstract}

Background: Process evaluations of randomized controlled trials (RCTs) can provide insight and inform us on the intervention implementation, causal mechanisms and the contextual factors; whether an intervention is effective or ineffective due to implementation failure or failure of the intervention itself. Hence, we aim to consolidate the methodology from previous process evaluations of complex interventions in healthcare services upon their findings on facilitators and barriers to address the prevention of type 2 diabetes among women with gestational diabetes mellitus (GDM).

Methods: Comprehensive search will be conducted on electronic databases, trial registers, and reference lists of recent reviews for RCTs of complex interventions which address process evaluations of diabetes prevention intervention (DPI) for women with GDM in healthcare settings. There are no restrictions on the year of publication and language of the papers. Data from each study will be extracted by two reviewers independently using standardized forms. Data extracted include descriptive items on the study design and the outcomes of process evaluations from the three dimensions: (1) implementation (theoretical framework, reach, dose delivered, dose received, fidelity, behaviour change techniques and competency in delivering them); (2) mechanistic (format of delivery either digital, non-digital or hybrid, participant and healthcare provider experience and feedback; (3) contextual (socio-cultural context, the organisational factors, local and national policy factors as barriers, facilitators, contamination and adoption for the DPI and the control as well as the conflict of interest). The quality of the studies will be assessed using mixed methods appraisal tool (MMAT) which designed for the appraisal stage of systematic mixed studies reviews. A narrative and thematic synthesis of the findings will be presented to inform the contents of a new DPI for women with GDM.

Discussion: The findings from this process evaluation findings are valuable in determining 
whether a complex intervention should be scaled up or modified for other contexts in future plan. Publishing this protocol could benefit our understanding on how the process evaluation have been conducted thus far and give deeper input in understanding potential challenges and solutions to aid in the implementation of effective DPIs for GDM in Malaysia.

Systematic Review Registration: CRD42020208212

Keywords: Process evaluations, gestational diabetes mellitus, randomized controlled trial, diabetes prevention, lifestyle intervention, maternal outcomes, and antenatal outcomes. 


\section{BACKGROUND}

Gestational diabetes mellitus (GDM) is a condition that occurs when there is impairment in glucose tolerance during pregnancy, with $84 \%$ of diabetes in pregnancy being GDM[1]. The worldwide prevalence of GDM ranged from 8 - 27\% in 2019 with the incidence of GDM continuing to increase, especially in low to middle income countries[1]. For example, 1 in 4 live births in South East Africa were affected by GDM compared to 1 in 6 live births in Europe. In Malaysia, the prevalence of GDM ranges 8 - 28\% [1-3]. The maternal consequences of GDM are wide ranging including obstetric complications such as preeclampsia and a 2-7 fold increased risk of having future episodes of GDM and developing type 2 diabetes (T2D)[4-5] and increased risk of cardiovascular diseases[6]. For instance, the International Hyperglycaemia and Adverse Pregnancy Outcomes (HAPO) study found that around half of the women with GDM developed T2D or prediabetes 11 years later[7]. The risk factors for this conversion include maternal age, glucose levels in pregnancy, family history of diabetes, pre-pregnancy and postpartum body mass index, dietary patterns, physical activity, breastfeeding and genetic predisposition[8-10]. Women with GDM are also more likely to have antenatal and postnatal depression than those without GDM[11-12].

There are also potential adverse outcomes for the infant such as shoulder dystocia, neonatal hyperbilirubinaemia, hypoglycemia and respiratory distress syndrome[13] in the short term and increased risk of T2D in the long term[14].

As women receive more medical attention during the antenatal and postpartum period than any other stage in their life course, this represents an important window of opportunity for prevention of T2D post-GDM. While randomised controlled trials (RCT) have shown that intensive DPIs are more effective in reducing T2D risk than usual care in the general population[15-17], RCTs of DPIs for women with GDM have to date been less effective in 
preventing future GDM episodes or T2D. There have been approximately 30 such RCTs in the past 10 years. A recent meta-analysis in women with GDM reported mixed results; of the 15 RCTs included in the review, in 8 studies that could be pooled, there was a non-significant reduction in risk for $\mathrm{T} 2 \mathrm{D}$ (risk ratio $=0.75,95 \%$ confidence interval $(\mathrm{CI})$ 0.55-1.03[18]. More recent RCTs have reported a small non-significant mean reduction in weight over 12 months[19] and a non-significant reduction in T2D at 3 years[20].

There is a need to understand why current models of DPI in GDM women are of limited effectiveness. As DPIs are, in effect, complex interventions made up of multiple components or active ingredients[21], one approach could be is to critique the process evaluation[22]. This is an umbrella term for a range of theoretical constructs and methodologies that aim to identify the key components of a complex intervention that help explain how and why they are (or are not) effective.

The Medical Research Council (MRC) have produced a theoretical framework that describes three dimensions of a process evaluation (see Figure 1). First there is implementation which is the process through which interventions are delivered, and what is delivered in practice. These can include the key structures, the theoretical framework, resources, mechanisms through which delivery is achieved; the fidelity such as the consistency of what is implemented with the planned intervention; the dose to define how much of an intervention is delivered; and reach which refers to the extent to which a target audience comes into contact with the intervention. Second are the mechanisms that aim to explain the pathway through which intervention activities produce intended (or unintended) effects. The study of mechanisms may include participant and therapist responses to interaction with a complex intervention and examining mediating or intermediate processes which explain subsequent changes in outcomes, including those unintended. Third is the context in which the 
intervention is set. This includes moderating factors external to the intervention but that may influence its implementation, such as health policy, organisational factors or changes in delivery of medical care, socio-cultural factors such as stigma or cultural values, lack of support to attend the complex intervention, and psychological factors such as shame or health beliefs. Process evaluations use quantitative and/or qualitative methods to examine these three dimensions (implementation, mechanisms of action and contextual factors) will help to identify the active ingredients in the RCTs[23].

Figure 1 Conceptual Framework

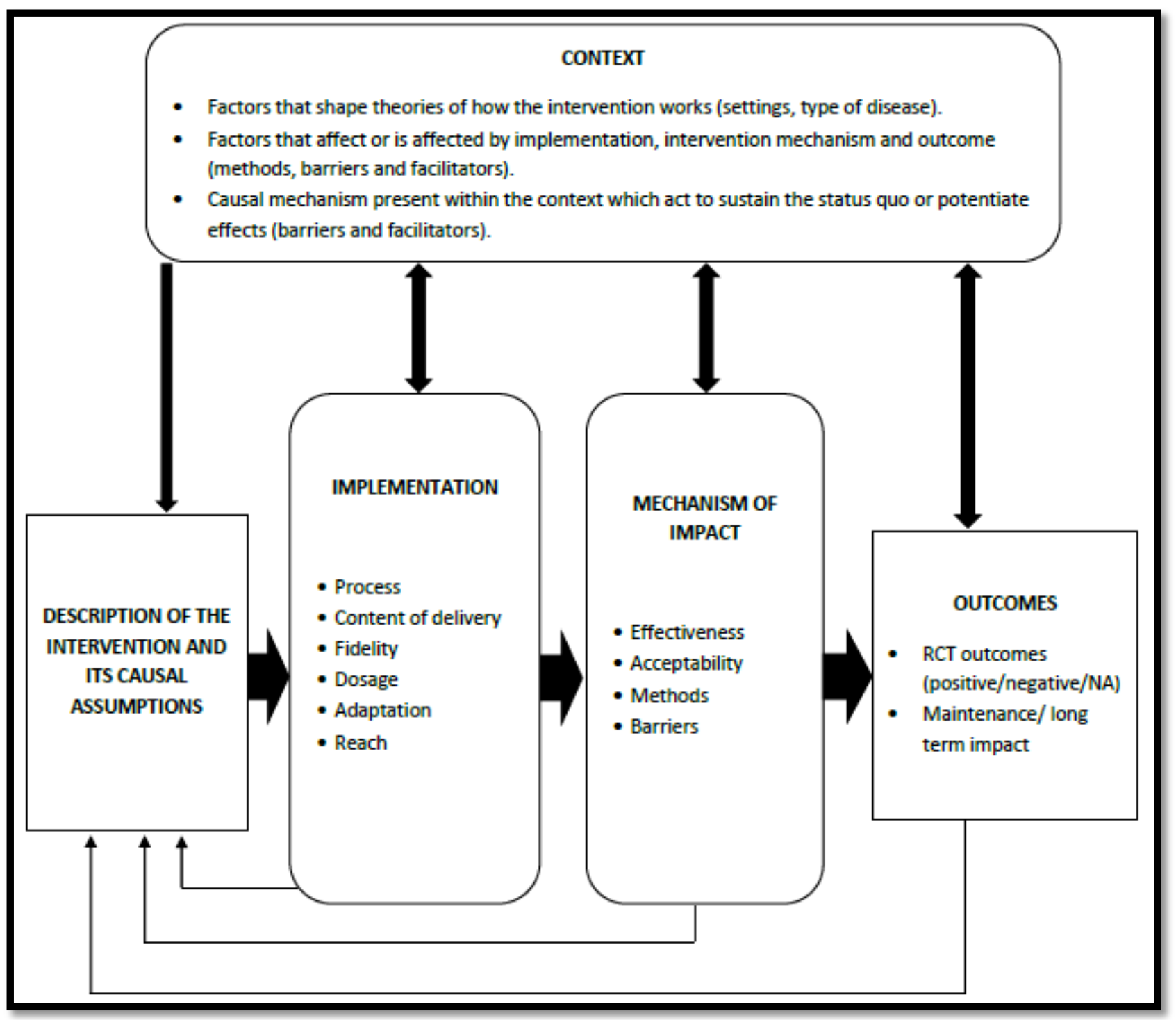

(Adapted from MRC Guidance on Process Evaluations by Moore et al., 2014) 
There has been no systematic review of process evaluation in RCTs of DPI in the healthcare settings and general population. However, processes that are most commonly reported in these DPIs often include nutritional advice and/or physical activity; behaviour change techniques such as goal setting and motivational interviewing; target setting such as aiming for $5-7 \%$ weight loss; specialist health care providers such as health trainers; 'dose' in terms of number of sessions and duration of the intervention; pacing of sessions such as initial high frequency of sessions followed by less frequent maintenance phases. In contrast, the majority of the DPI processes for GDM included diet and physical advice[18]. There was tentative suggestion that those DPIs which started soon after delivery were more effective (risk ratio = 0.61, 95\%CI 0.40-0.94). There have been less than a handful of RCTs that have used breastfeeding as an intervention.

This study therefore first aims to conduct a systematic review of the processes evaluated in RCTs of DPIs in GDM; second to describe the range of processes assessed and the methods used to do so; third to identify processes that overall appear to be a) more effective and b) not effective in reducing the risk of T2D post GDM. These findings will inform the content of a DPI for women with GDM in Malaysia. 


\section{METHODOLOGY}

\section{Design and registration}

We will conduct the systematic review in accordance with Preferred Reporting Items for Systematic reviews and Meta-Analyses Protocols (PRISMA-P) guidelines and checklist (see appendix). The systematic review is prospectively registered with PROSPERO (Registration number: CRD42020208212) and amendments will be documented with version description.

\section{Eligibility criteria}

1. Types of studies

Only RCTs will be included; cohort and uncontrolled before-and-after studies and case series, reviews, non-journal article, non-report based on empirical research and non-human research will be excluded. We will aim to extract process evaluation findings from all RCTs whether or not these have been explicitly described as some RCTs do include some aspects of process evaluation.

\section{Types of patients}

The patient group will consist of women who have current or a history of GDM. Women at high risk of GDM such as those with obesity, family history of T2D, menopausal and postmenopausal women will be excluded.

3. Types of interventions

The interventions included will be complex interventions consisting of lifestyle change delivered either in the antenatal and/or postnatal period through digitalised, faceto-face or mixed methods intervention during the antenatal and/or postnatal period. It includes dietary management, physical activity, psychological aspect and others. Interventions that test a single ingredient e.g. a pharmacological agent will be excluded. 


\section{The comparators}

It may include treatment as usual, active control or placebo control. Placebo controlled is a condition where one that appears in all respects to be identical to the treatment condition and lacking on the critical ingredient of the treatment. Meanwhile, an active control is one in which participants engage in some task during the intervention period.

\section{Types of outcomes}

The primary outcome will be the strengths and limitations of each process evaluation as well as the identification of implementation barriers and facilitators for the complex intervention following MRC guidance as a reference point. Quantitative and qualitative data will be extracted, coded and grouped into categories of implementation, mechanism and context dimensions and their related constructs. In the implementation dimension the following constructs will be assessed: the theoretical framework used to inform the intervention; the outreach to the extent the target audience come into contact with the intervention; dose (number of sessions or total time) delivered; dose received; constancy of delivering protocol; description of behaviour change techniques and competency in delivering them. In the mechanistic dimension, the following will be extracted: format of delivery (digital, nondigital or hybrid); participant experience; health provider experience. Whereas in the context dimension, we will extract data on socio-cultural context; the organisational factors; local and national policy factors (as barriers, facilitators, contamination and adoption) for the DPI and the control. Inductive derivation of the key themes was done through constant comparison between the findings from the papers within each category and examining the relationships between them. The secondary outcomes we will extract include: maternal rates of T2D and of subsequent episodes of GDM; risk factor for T2D (weight, lipid profile); psychological issue; mother-infant relationship; maternal complications; and infant complications. 


\section{Information sources}

We will search from the following databases: OVID (EMBASE, MEDLINE, PsycINFO, Global Health); Cochrane Library, PUBMED and hand-searching of reference lists of recent reviews[18,24]. For each RCT included in this review, we will specifically search for duplicates and secondary publications, and contacting all the authors to request any published or unpublished process evaluation. Forward and backward citation will also be used to screen for eligible articles.

\section{Search strategy}

The keywords will be developed and iteratively refined from the research questions and definitions of key concepts by using Cochrane search terms for diabetes prevention, gestational diabetes mellitus and lifestyle interventions including antenatal, postnatal, breastfeeding, type 2 diabetes, randomised control trial and process evaluations. The main key words used will be gestational diabetes mellitus, randomised controlled trial and process evaluation. Boolean search will be used to combine the keywords with operators such as AND, NOT and OR to further produce more relevant results. For example: ((Gestational diabetes mellitus OR gestational diabetes OR pregnancy induced diabetes OR diabetes in pregnancy) AND ((Randomized controlled trial OR controlled clinical trial OR pragmatic control trial OR clinical trial) AND (process evaluation OR program evaluation OR process assessment OR process acceptance OR outcome measures)); ((Gestational diabetes mellitus OR gestational diabetes OR pregnancy induce diabetes )) AND ((Randomized Controlled Trial OR controlled clinical trial OR pragmatic clinical trial OR clinical trial) OR (process evaluation OR program assessment OR process acceptance OR outcome measures) 


\section{Data management}

Rayyan QCRI will be used for title and abstract screening. Duplicate papers will be identified and removed accordingly from search strategy database and Endnote. Two authors will independently assess the eligibility of the publications based first on the titles and abstracts of records identified by the searches. After excluding papers that do not meet eligibility criteria based on titles and abstracts, the remaining publications will be downloaded for further assessment of eligibility. Data will be stored in a common file which will be password protected. Non-English language papers will be translated into English by bi-lingual professionals or academics. The reviewers will resolve disagreements as to eligibility through a consensus-based discussion, and if necessary, with a third reviewer.

\section{Standardised data extraction}

The following data about the RCT will be extracted: general characteristic of the study (author, year of publication, studies country, sample size, type of intervention and socioeconomic status); participant's characteristics (age, ethnicity, marital status, parity, type of birth, education and employment); duration of follow up; and the effect sizes of the main outcome(s). The outcomes we will extract include: maternal rates of T2D and of subsequent episodes of GDM; risk factor for T2D (weight, lipids profile); psychological issue (depression); mother-infant relationship; other maternal and infant complications.

In terms of the process evaluation aspects, the MRC provides many examples of processes but there is no consensus for a gold standard or a minimum checklist for process evaluation. Therefore, we have derived a checklist of process constructs within the three dimensions. Qualitative and quantitative data will be extracted for each construct. In the implementation dimension the following constructs will be assessed: the theoretical framework used to inform the intervention; an assessment of reach; dose (number of sessions or total time) 
delivered; dose received; fidelity of delivering protocol as stated; description of behaviour change techniques and competency in delivering them. In the mechanistic dimension, the following will be extracted: format of delivery (digital, non-digital or hybrid); participant experience; health provider experience. Digital interventions will include the use of telephone, video-conferencing, mobile short message service (SMS), apps or web-based interfaces and other remote wireless relay systems[22]. In the contextual factors, we will extract data on socio-cultural context; the organisational factors; local and national policy factors (as barriers, facilitators, contamination and adoption) for the DPI and the control. The data extraction forms will be pilot tested by the two reviewers and iteratively revised. Two reviewers will independently extract data from the rest of the included list of articles.

\section{Quality assessment}

Data to assess quality will be extracted from the included studies. Quality assessment tool or risk of bias tool use scale or checklist which provides a list of criteria or domain in order to appraise quality of the study. Hence, a unique tool known as mixed method appraisal tool (MMAT), 2018 will be used to assess the quality of different study designs. This tool is about judgment making by the reviewers. For each included study, it is necessary for the reviewer to choose appropriate category of studies to appraise. Then, rate the criteria of the chosen category accordingly. The 'Can't tell' response category indicates that the paper do not report appropriate information to answer 'Yes' or 'No' and the reviewer would look for companion papers or contact the authors for more information or clarification. In terms of scoring, performing a sensitivity analysis by contrasting the results between the studies to better inform the quality of the included studies. 
Figure 2 Mixed Methods Appraisal Tools (MMAT), 2018

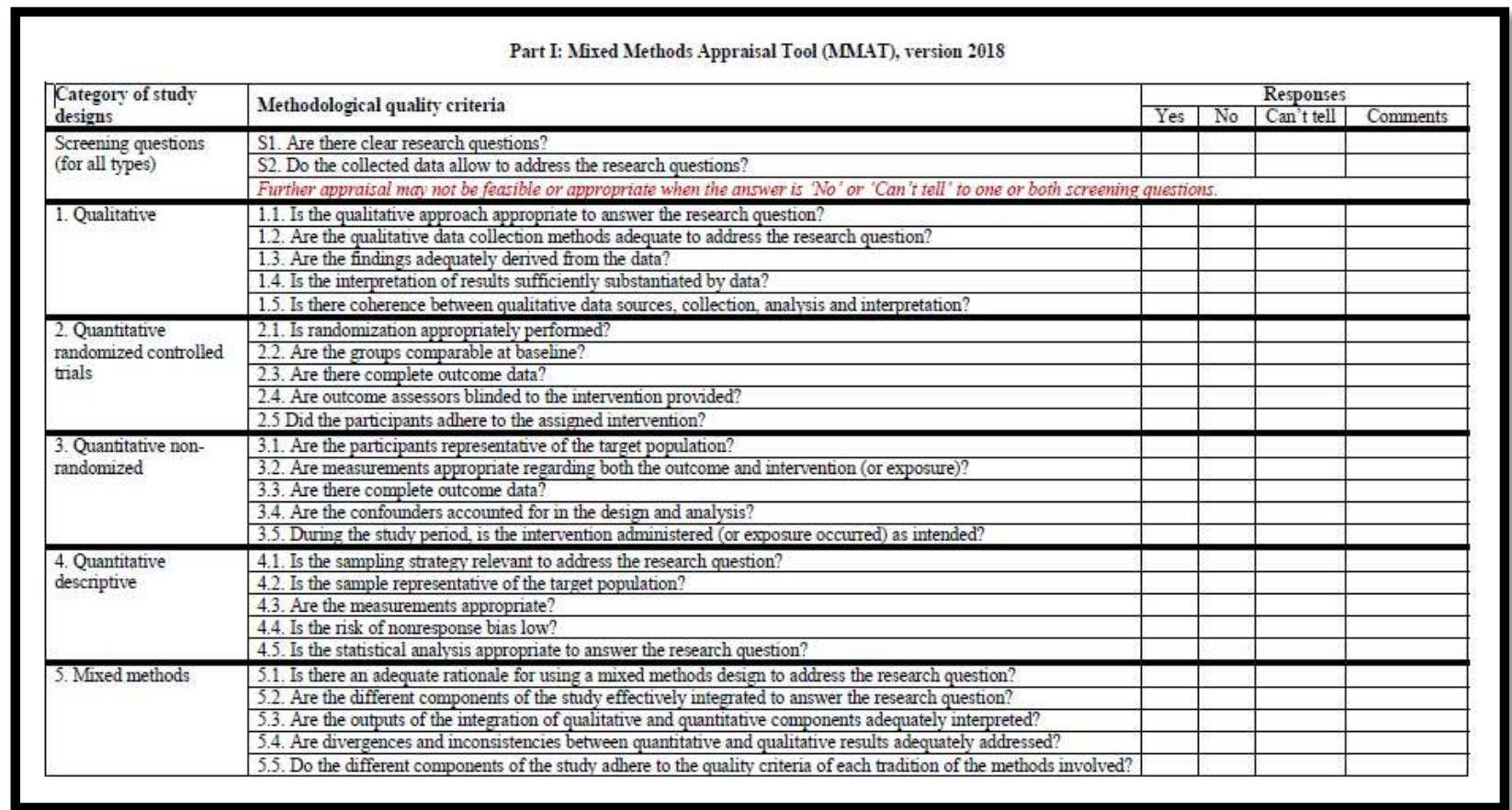

\section{Data synthesis and outputs}

The extracted qualitative and quantitative data for each of the three MRC process evaluation dimensions will be summarised. In quantitative part, descriptive items (ie: number of positive RCTs) will be tallied and synthesised into three tables: (1) Overall characteristics of the study; (2) methodology: stages of the process evaluation will be gathered and grouped among the studies grouping (ie, feasibility/piloting, effectiveness, post-evaluation) and (3) quality assessment: using a tool developed by the Effective Public Health Practice Project (EPHPP) for knowledge synthesis which provides a standardized means to assess study quality and overall methodological rating of either strong, moderate or weak category for evidence support practice. Meanwhile for the qualitative part, the extracted data will be coded and grouped into categories of implementation, mechanism and context. The key themes derivation will be identified through reasoning and constant comparison between the findings from the collective papers within each category by examining the relationships between them. Using a modified MRC framework, we will map our methodological and 
implementation findings to triangulate and synthesise the data. Triangulation takes part as a method to develop comprehensive understanding and to act as a strategy to test the validity through the convergence of information from different sources of the data. These categories of data information will then be subjected to thematic synthesis in order to produce a comprehensive set of synthesised findings[25]. Throughout the review, patient and public perspectives are synthesised from published papers and hence no public and patients were directly involved in this study. The findings will be synthesised according to each aim and will be used to inform the contents of a new DPI for women with GDM in Malaysia. 
FIGURE 3: Preferred Reporting Items for systematic review and meta-analyses (PRISMA): RCT randomized controlled trial.

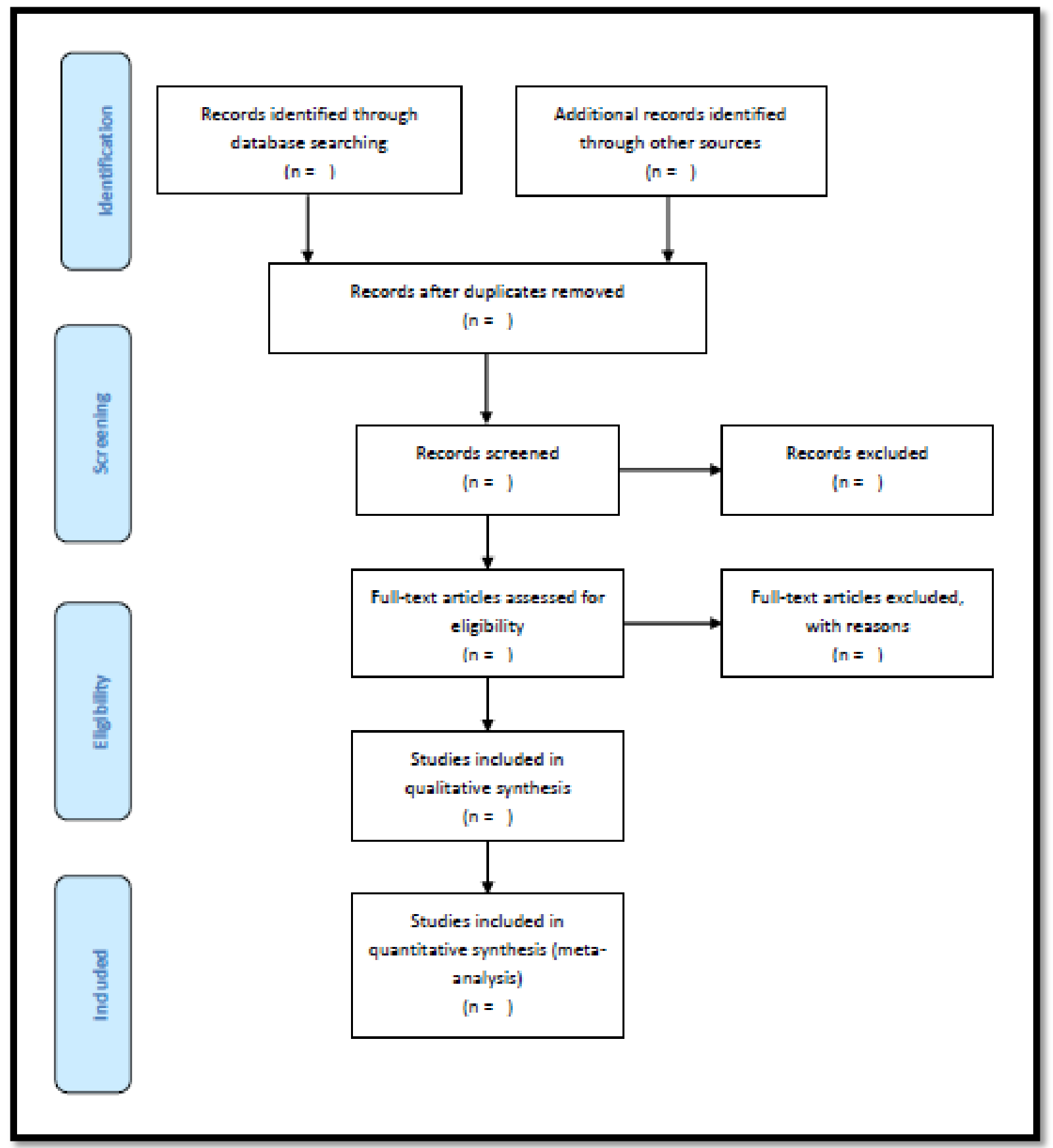




\section{DISCUSSION}

There have been approximately 30 RCTs of complex lifestyle interventions to reduce the risk of T2D in women with GDM yet collectively the evidence for the effectiveness of these interventions remains limited. Before conducting another RCT of DPI in this high-risk group, it is important to understand why previous RCTs have either had negative or weak effects. Process evaluation integrated into RCTs of complex interventions capture an umbrella of concepts to help explain the underlying mechanisms of action. They vary in their objectives, methodology and the selection of outcome and there is no consensus as yet as to how best to conduct them. We have proposed a framework for a systematic review that will extract and summarise what processes have been evaluated under the three key MRC dimensions of implementation, mechanism and context. We will use the findings to inform the development of a logic model, the contents of a digital app, and derive a process evaluation framework for our future feasibility study testing of a refined DPI.

The strengths of this protocol include that it sets out a priori a systematic review using a comprehensive approach to include all RCTs regardless of whether the term process evaluation is explicitly described. As there is no validated consensus checklist to conduct a process evaluation, we have adapted other models[26-27]. We have proposed a pragmatic checklist of key processes using widely accepted current theories and national guidance[22].

The limitations are that we may have unintentionally omitted one or more relevant processes but we aim to reduce this risk by piloting the data extraction schedule with several RCTs and a priori revising the protocol if new processes are identified during the review. There are some frameworks we could have used instead such as the Wagner Chronic Care model which six interdependent elements of a complex intervention namely the Health System: The Community: Self-Management Support: Decision Support: Delivery System 
Design: and Clinical Information Systems but they do not give guidance on the methods for assessing the processes[28].

\section{CONCLUSION}

In summary, we have devised a protocol for a systematic review of process evaluations for DPI for GDM and the findings are potentially informative for other lifestyle interventions to reduce chronic diseases such as obesity, cardiovascular diseases and depression. 


\section{LIST OF ABBREVIATION}

GDM: gestational diabetes mellitus

DPI: diabetes prevention intervention

PE: process evaluation

T2D: type 2 diabetes

RCT: randomized controlled trial

MMAT: mixed method appraisal tool

SMS: mobile short message service 


\section{DECLARATIONS}

\section{Ethics approval and consent to participate}

Not applicable

\section{Consent for publication}

Not applicable

\section{Availability of data and materials}

Not applicable

\section{Competing interests}

The authors declare that have no competing interests.

\section{Funding}

This work is supported by MYPAIR Grant UK-Malaysia: Joint Partnership Call on NonCommunicable Diseases (Malaysia: JPT.S(BPKl)2000/011/06/05 (27); UK: MR/T018240/1).

\section{Author's contribution}

$\mathrm{KI}$ and BHC designed the review. IIMS lead and the drafting of the protocol. IPN, AF, KG and MP provided input on the search strategies. BHC, KI, IPN, AF, KG, IZI and FH provided substantive feedback on the manuscript. All authors contributed to the manuscript drafts and reviewed the final manuscript. All authors approved the final protocol.

\section{Acknowledgements}

We would like to thank MYPAIR Grant UK-Malaysia for providing the funding for the systemic review. We would like to thank our Knowledge User Advisory Panel members for providing guidance and feedback. Their contributions represent independent research part funded by the NIHR Biomedical Research Centre (South London and Maudsley NHS Foundation Trust and KCL) and the NIHR Applied Research Collaboration South London (King's College Hospital NHS Foundation Trust). The views expressed are those of the author(s) and not necessarily those of the NHS, the NIHR or the Department of Health and Social Care. MYGODDESS Project team members and collaborators include Dr. Hanifatiyah 
Ali from the Department of Family Medicine Universiti Putra Malaysia who helped in the early protocol drafting by IIMS, MSc student Ms. Pamela Phui Har Yap who, Dr. Ziti Akthar Supian at Seri Kembangan Health Clinic, Dr. Haslinda Hassan at Puchong Health Clinic, Dr. Fuziah Paimin at Putrajaya Presint 9 Health Clinic, Dr. Nurain Mohd. Noor and Dr. Wan Ahmad Hazim Wan Ghazali from the Departments of Medicine and Obstetrics and Gynaecology in Hospital Putrajaya, respectively. 


\section{REFERENCES}

1. International Diabetes Atlas, 2019. Available from: URL:

https://www.diabetesatlas.org/en/

2. $4^{\text {th }}$ Report of National Obstetrics Surgery, 2013-2015, Malaysia. Available from:

URL: http://www.acrm.org.my/nor/doc/reports/17052018_nor_report.pdf

3. Logakodie S, Azahadi O, Fuziah P, Norizzati BI, Tan SF, Zienna ZZ, Norliza M, Noraini J, Hazlin M, Noraliza MZ, Sazidah MK. Gestational diabetes mellitus: The prevalence, associated factors and foeto-maternal outcome of women attending antenatal care. Malaysian family physician: the official journal of the Academy of Family Physicians of Malaysia. 2017;12(2):9. Available from: URL: https://www.ncbi.nlm.nih.gov/pmc/articles/PMC5802775/

4. Bellamy L, Casas JP, Hingorani AD, Williams D. Type 2 diabetes mellitus after gestational diabetes: a systematic review and meta-analysis. The Lancet. 2009 May 23;373(9677):1773-9. Available from: URL: https://www.sciencedirect.com/science/article/pii/S0140673609607315

5. Reece EA. The fetal and maternal consequences of gestational diabetes mellitus. The journal of maternal-fetal \& neonatal medicine. 2010 Mar 1;23(3):199-203. Available from: URL: https://www.tandfonline.com/doi/abs/10.3109/14767050903550659

6. Lekva T, Bollerslev J, Norwitz ER, Aukrust P, Henriksen T, Ueland T. Aortic stiffness and cardiovascular risk in women with previous gestational diabetes mellitus. PloS one. 2015 Aug 26;10(8):e0136892. Available from: URL: https://journals.plos.org/plosone/article?id=10.1371/journal.pone.0136892

7. HAPO Study Cooperative Research Group. Hyperglycaemia and Adverse Pregnancy Outcome (HAPO) Study: associations with maternal body mass index. BJOG: An International Journal of Obstetrics \& Gynaecology. 2010 Apr;117(5):575-84. 
Available from: URL: https://obgyn.onlinelibrary.wiley.com/doi/full/10.1111/j.1471$\underline{0528.2009 .02486 . x}$

8. Liu H, Zhang C, Zhang S, Wang L, Leng J, Liu D, Fang H, Li W, Yu Z, Yang X, Dong L. Prepregnancy body mass index and weight change on postpartum diabetes risk among gestational diabetes women. Obesity. 2014 Jun;22(6):1560-7. Available from: URL: https://onlinelibrary.wiley.com/doi/full/10.1002/oby.20722

9. Gunderson EP, Hurston SR, Ning X, Lo JC, Crites Y, Walton D, Dewey KG, Azevedo RA, Young S, Fox G, Elmasian CC. Lactation and progression to type 2 diabetes mellitus after gestational diabetes mellitus: a prospective cohort study. Annals of internal medicine. 2015 Dec 15;163(12):889-98. Available from: URL: https://www.acpjournals.org/doi/abs/10.7326/M15-0807

10. Davis JN, Shearrer GE, Tao W, Hurston SR, Gunderson EP. Dietary variables associated with substantial postpartum weight retention at 1-year among women with GDM pregnancy. BMC obesity. 2017 Dec 1;4(1):31. Available from: https://link.springer.com/article/10.1186/s40608-017-0166-0

11. Napoli A, Lamis DA, Berardelli I, Canzonetta V, Sarubbi S, Rogante E, Napoli PL, Serafini G, Erbuto D, Tambelli R, Amore M. Anxiety, Prenatal Attachment, and Depressive Symptoms in Women with Diabetes in Pregnancy. International Journal of Environmental Research and Public Health. 2020 Jan;17(2):425. Available from: URL: https://www.mdpi.com/1660-4601/17/2/425

12. Wilson CA, Santorelli G, Dickerson J, Ismail K, Reynolds RM, Simonoff E, Howard LM. Is there an association between anxiety and depression prior to and during pregnancy and gestational diabetes? An analysis of the Born in Bradford cohort. Journal of Affective Disorders. 2020 Nov 1;276:345-50. Available from: URL: https://www.sciencedirect.com/science/article/pii/S0165032720324241 
13. Bhandiwad A, Divyasree B, Gowda SL. Adverse maternal and perinatal outcomes in gestational diabetes mellitus. International Journal of Medical Research \& Health Sciences. 2015;4(4):775-7. Available from: URL:

https://www.researchgate.net/profile/Surakshith_Gowda/publication/283787874_Adv erse_maternal_and_perinatal_outcomes_in_gestational_diabetes_mellitus/links/575d8 b3b08ae9a9c955a6dd6/Adverse-maternal-and-perinatal-outcomes-in-gestationaldiabetes-mellitus.pdf

14. Lowe WL, Scholtens DM, Lowe LP, Kuang A, Nodzenski M, Talbot O, Catalano PM, Linder B, Brickman WJ, Clayton P, Deerochanawong C. Association of gestational diabetes with maternal disorders of glucose metabolism and childhood adiposity. Jama. 2018 Sep 11;320(10):1005-16. Available from: https://jamanetwork.com/journals/jama/article-abstract/2702144

15. Diabetes Prevention Program Research Group. Long-term effects of lifestyle intervention or metformin on diabetes development and microvascular complications over 15-year follow-up: the Diabetes Prevention Program Outcomes Study. The lancet Diabetes \& endocrinology. 2015 Nov 1;3(11):866-75. Available from: URL: https://www.sciencedirect.com/science/article/abs/pii/S2213858715002910

16. Tuomilehto J, Lindström J, Eriksson JG, Valle TT, Hämäläinen H, Ilanne-Parikka P, Keinänen-Kiukaanniemi S, Laakso M, Louheranta A, Rastas M, Salminen V. Prevention of type 2 diabetes mellitus by changes in lifestyle among subjects with impaired glucose tolerance. New England Journal of Medicine. 2001 May 3;344(18):1343-50. Availbale from: URL: https://www.nejm.org/doi/full/10.1056/nejm200105033441801

17. Gong Q, Zhang P, Wang J, Ma J, An Y, Chen Y, Zhang B, Feng X, Li H, Chen X, Cheng YJ. Morbidity and mortality after lifestyle intervention for people with impaired glucose tolerance: 30-year results of the Da Qing Diabetes Prevention 
Outcome Study. The Lancet Diabetes \& Endocrinology. 2019 Jun 1;7(6):452-61. Available from: URL:

https://www.sciencedirect.com/science/article/abs/pii/S2213858719300932

18. Goveia P, Cañon-Montañez W, Santos DD, Lopes GW, Ma RC, Duncan BB, Ziegelman PK, Schmidt MI. Lifestyle intervention for the prevention of diabetes in women with previous gestational diabetes mellitus: a systematic review and metaanalysis. Frontiers in endocrinology. 2018 Oct 5;9:583. Available from: URL: https://www.frontiersin.org/articles/10.3389/fendo.2018.00583/full

19. Liu H, Wang L, Zhang S, Leng J, Li N, Li W, Wang J, Tian H, Qi L, Yang X, Yu Z. One-year weight losses in the Tianjin Gestational Diabetes Mellitus Prevention Programme: A randomized clinical trial. Diabetes, obesity and metabolism. 2018 May;20(5):1246-55. Available from: URL: https://dompubs.onlinelibrary.wiley.com/doi/full/10.1111/dom.13225?casa token=Xtom5iWjZd oAAAAA\%3AFmy-jefxCIBvlAdcurLMDildPR2y6zSgPgBId07A0K6Uwjt$\underline{\text { hYzcXBNsYa_3R4fakddCUSwnyDUAk_dK }}$

20. Shek NW, Ngai CS, Lee CP, Chan JY, Lao TT. Lifestyle modifications in the development of diabetes mellitus and metabolic syndrome in Chinese women who had gestational diabetes mellitus: a randomized interventional trial. Archives of gynecology and obstetrics. 2014 Feb 1;289(2):319-27. Available from: URL: https://link.springer.com/article/10.1007/s00404-013-2971-0

21. Craig P, Dieppe P, Macintyre S, Michie S, Nazareth I, Petticrew M. Developing and evaluating complex interventions: the new Medical Research Council guidance. Bmj. 2008 Sep 29;337. Available from: https://www.bmj.com/content/337/bmj.a1655.short 
22. Moore GF, Audrey S, Barker M, Bond L, Bonell C, Hardeman W, Moore L, O'Cathain A, Tinati T, Wight D, Baird J. Process evaluation of complex interventions: Medical Research Council guidance. bmj. 2015 Mar 19;350. Available from: https://www.bmj.com/content/350/bmj.h1258/

23. Anderson LM, Oliver SR, Michie S, Rehfuess E, Noyes J, Shemilt I. Investigating complexity in systematic reviews of interventions by using a spectrum of methods. Journal of clinical epidemiology. 2013 Nov 1;66(11):1223-9. Available from: https://www.sciencedirect.com/science/article/pii/S0895435613002576?casa token= $\underline{\text { KUXUmRsW8 EAAAAA:iLRIYWpN4-VTaSz3FCScR5VATR- }}$ 41COiAmynR8FA9RBDAHjZ99c1MlN5ppM8Thh6XhmQHHqc6pU

24. Miyazaki C, Tanase-Nakao K, Arata N, Mori R, Kawasaki M, Ota E. Nonpharmacological interventions to prevent type 2 diabetes in women diagnosed with gestational diabetes mellitus: a systematic overview. Diabetology international. 2017 Jun 1;8(2):160-80. Available from: https://link.springer.com/article/10.1007\%2Fs13340-017-0316-0

25. Tong A, Flemming K, McInnes E, Oliver S, Craig J. Enhancing transparency in reporting the synthesis of qualitative research: ENTREQ. BMC medical research methodology. 2012 Dec;12(1):181. Available from: URL: https://bmcmedresmethodol.biomedcentral.com/articles/10.1186/1471-2288-12$\underline{181 \text { ?optIn=false }}$

26. Saunders M. The presence of evaluation theory and practice in educational and social development: toward an inclusive approach. London Review of Education. 2006 Jul 1;4(2):197-215. Available from: URL:

https://www.ingentaconnect.com/content/ioep/clre/2006/00000004/00000002/art0000 $\underline{7}$ 
27. Grant A, Treweek S, Dreischulte T, Foy R, Guthrie B. Process evaluations for clusterrandomised trials of complex interventions: a proposed framework for design and reporting. Trials. 2013 Dec 1;14(1):15. Available from: https://link.springer.com/article/10.1186/1745-6215-14-15

28. Barr V, Robinson S, Marin-Link B, Underhill L, Dotts A, Ravensdale D, Salivaras S. The expanded chronic care model. Hosp Q. 2003;7(1):73-82. Available from: URL: https://www.area-c54.it/public/the\%20expanded\%20chronic\%20care\%20model.pdf 


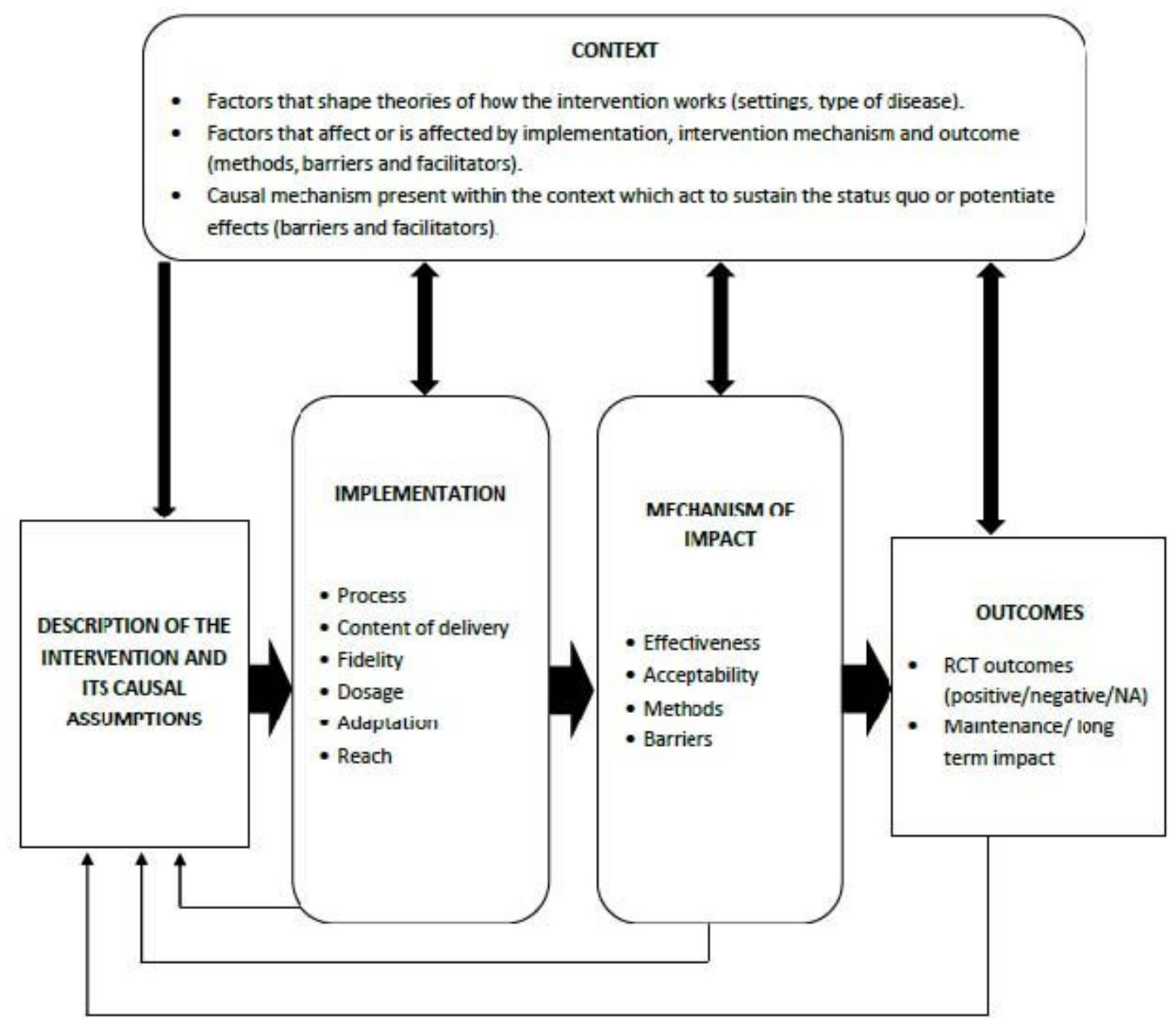

Figure 1

Conceptual Framework (Adapted from MRC Guidance on Process Evaluations by Moore et al., 2014) 
Part I: Mixed Methods Appraisal Tool (MMAT), version 2018

\begin{tabular}{|c|c|c|c|c|c|}
\hline \multirow{2}{*}{$\begin{array}{l}\text { Category of study } \\
\text { desigus }\end{array}$} & \multirow{2}{*}{ Methodological quality criteria } & \multicolumn{4}{|c|}{ Responses } \\
\hline & & Yes & No & Can't tell & Comments \\
\hline \multirow{3}{*}{$\begin{array}{l}\text { Screening questions } \\
\text { (for all types) }\end{array}$} & S1. Are there clear research questions? & & & & \\
\hline & S2. Do the collected data allow to address the research questions? & & & & \\
\hline & Further appraisal mav not be feasible or appropriate when the answer is ' $\mathrm{No}$ ' or 'Can't tell' to one or both screening & questio & & & \\
\hline \multirow{5}{*}{ 1. Qualitative } & 1.1. Is the qualitative approach appropriate to answer the research question? & & & & \\
\hline & 1.2. Are the qualitative data collection methods adequate to address the research question? & & & & \\
\hline & 1.3. Are the findings adequately derived from the data? & & & & \\
\hline & 1.4. Is the interpretation of results sufficiently substantiated by data? & & & & \\
\hline & 1.5. Is there coherence between qualitative data sources, collection analysis and interpretation? & & & & \\
\hline \multirow{5}{*}{$\begin{array}{l}\text { 2. Quantitative } \\
\text { randomized controlled } \\
\text { trials }\end{array}$} & 2.1. Is randomization appropriately performed? & & & & \\
\hline & 2.2. Are the groups comparable at baseline? & & & & \\
\hline & 2.3. Are there complete outcome data? & & & & \\
\hline & 2.4. Are outcome assessors blinded to the intervention provided? & & & & \\
\hline & 2.5 Did the participants adhere to the assigned intervention? & & & & \\
\hline \multirow{5}{*}{$\begin{array}{l}\text { 3. Quantitative non- } \\
\text { randomized }\end{array}$} & 3.1. Are the participants representative of the target population? & & & & \\
\hline & 3.2. Are measurements appropriate regarding both the outcome and intervention (or exposure)? & & & & \\
\hline & 3.3. Are there complete outcome data? & & & & \\
\hline & 3.4. Are the confounders accounted for in the design and analysis? & & & & \\
\hline & 3.5. During the study period, is the intervention administered (or exposure occurred) as intended? & & & & \\
\hline \multirow{5}{*}{$\begin{array}{l}\text { 4. Quantitative } \\
\text { descriptive }\end{array}$} & 4.1. Is the sampling strategy relevant to address the research question? & & & & \\
\hline & 4.2. Is the sample representative of the target population? & & & & \\
\hline & 4.3. Are the measurements appropriate? & & & & \\
\hline & 4.4. Is the risk of nonresponse bias low? & & & & \\
\hline & 4.5. Is the statistical analysis appropriate to answer the research question? & & & & \\
\hline \multirow[t]{5}{*}{ 5. Mixed methods } & 5.1. Is there an adequate rationale for using a mixed methods design to address the research question? & & & & \\
\hline & 5.2. Are the different components of the study effectively integrated to answer the research question? & & & & \\
\hline & 5.3. Are the outputs of the integration of qualitative and quantitative components adequately interpreted? & & & & \\
\hline & 5.4. Are divergences and inconsistencies between quantitative and qualitative results adequately addressed? & & & & \\
\hline & 5.5. Do the different components of the study adhere to the quality criteria of each tradition of the methods in & & & & \\
\hline
\end{tabular}

\section{Figure 2}

Mixed Methods Appraisal Tools (MMAT), 2018 


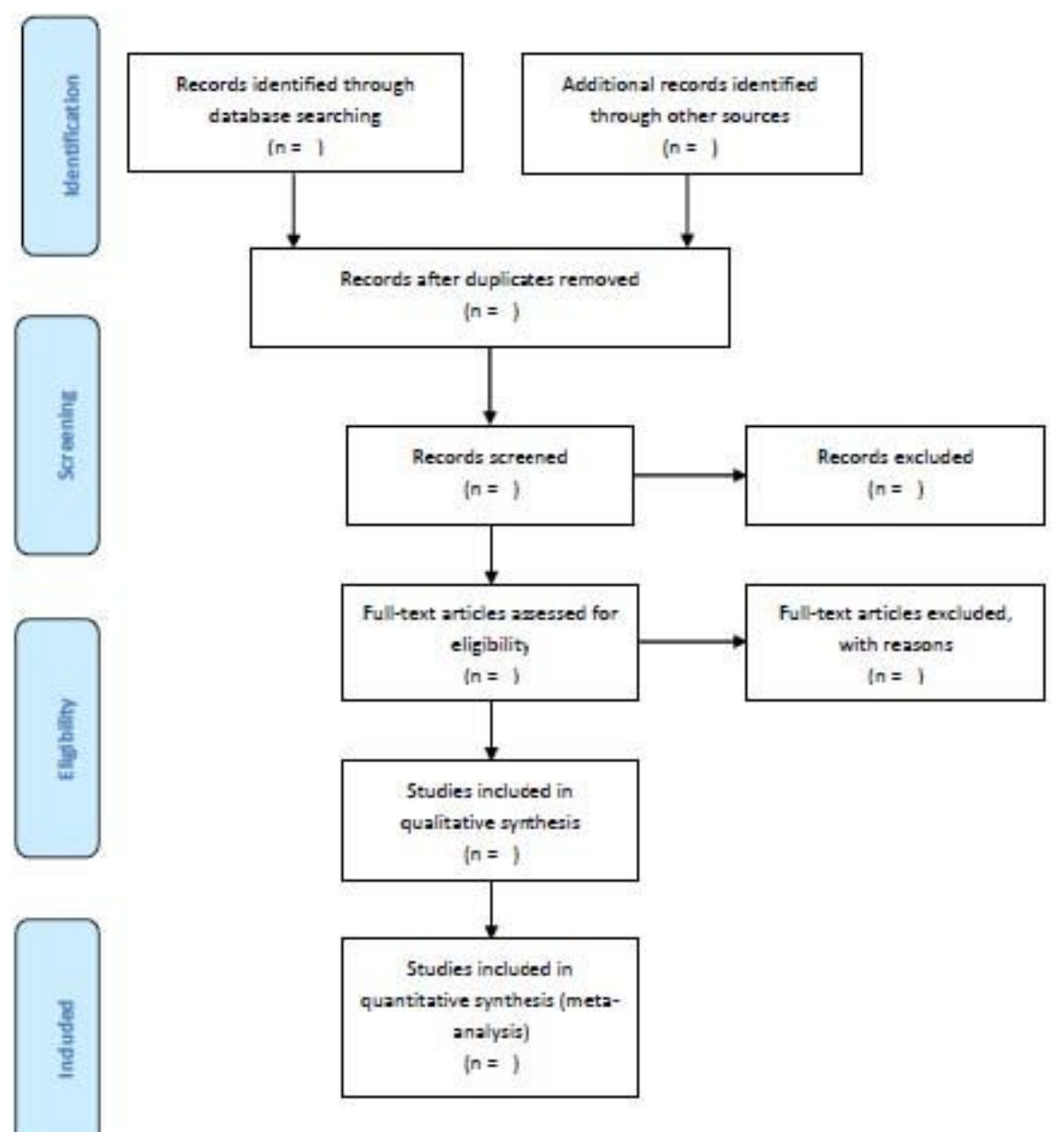

Figure 3

Preferred Reporting Items for systematic review and meta-analyses (PRISMA): RCT randomized controlled trial.

\section{Supplementary Files}

This is a list of supplementary files associated with this preprint. Click to download.

- DataretrievalformFinal.xlsx

- PRISMAPchecklistFinal.pdf 